\title{
Human peritoneal mesothelial cell transformation into myofibroblasts in response to TGF-B1 in vitro
}

\author{
ZHI-DONG LV, DI NA, XIAO-YANG MA, CHONG ZHAO, WEI-JUN ZHAO and HUI-MIAN XU \\ Department of Surgical Oncology, The First Affiliated Hospital, China Medical University, Shenyang 110001, P.R. China
}

Received August 22, 2010; Accepted October 13, 2010

DOI: $10.3892 / \mathrm{ijmm} .2010 .574$

\begin{abstract}
Peritoneal dissemination is one of the leading causes of death in gastric cancer patients. The interaction between carcinoma cells and the peritoneal lining may play a key role in tumor peritoneal dissemination. Human peritoneal mesothelial cells are a monolayer of squamous epithelial cells covering the peritoneal cavity and forming serosal membranes. The precise role of mesothelial cells in the peritoneal dissemination of gastric cancer remains to be identified. Expression of TGF-ß1, a cytokine known for its capacity to induce proliferative and transformative changes in cells, has been correlated with peritoneal metastasis and TNM stages of gastric cancer. High levels of TGF- $\beta 1$ in the subperitoneal milieu may play a key role in the transition of normal mesothelial cells to myofibroblasts. Here, we demonstrate that mesothelial cells activated by TGF- $\beta 1$ undergo epithelial-mesenchymal transition (EMT) and that the transition of mesothelial cells to myofibroblasts is dependent on Smad2 signaling. EMT of mesothelial cells was marked by up-regulation of $\alpha$-smooth muscle actin and vimentin expression. Cytokeratin and E-cadherin expression decreased over time in transformed mesothelial cells. Knockdown of Smad2 gene by siRNA silencing significantly suppressed the transition of mesothelial cells to myofibroblasts. We conclude that when exposed to TGF- $\beta 1$ mesothelial cells undergo EMT which involves Smad2 signaling. Furthermore, mesothelial cells may be the possible source of myofibroblasts in peritoneal fibrosis and provide a favorable environment for the dissemination of gastric cancer.
\end{abstract}

Correspondence to: Professor Hui-Mian Xu, Department of Surgical Oncology, The First Affiliated Hospital, China Medical University, Shenyang 110001, Liaoning Province, P.R. China E-mail: xuhuimianpf@163.com

Abbreviations: HPMCs, human peritoneal mesothelial cells; TGF- $\beta 1$, transforming growth factor- $\beta 1 ; \alpha$-SMA, $\alpha$-smooth muscle actin; ECM, extracellular matrix; EMT, epithelial-mesenchymal transition; $\mathrm{CK}$, cytokeratin

Key words: peritoneal fibrosis, peritoneal carcinomatosis, human peritoneal mesothelial cells, transforming growth factor- $\beta 1$, epithelial-mesenchymal transition

\section{Introduction}

Gastric cancer continues to be associated with high mortality. The poor outcome of this disease is to a large extent related to the metastatic spreading of cancer cells within the peritoneal cavity $(1,2)$. Attachment of malignant cells to the peritoneal mesothelium is thought to be a critical step in peritoneal dissemination of the disease. Available data indicate that the process is mediated by interactions between extracellular matrix (ECM) components produced by mesothelial cells and the corresponding adhesion molecules of cancer cells (3-5). Peritoneal fibrosis may provide a favorable environment for the dissemination of gastric cancer (6). The myofibroblast is believed to play a central role in the pathogenesis of peritoneal fibrosis. However, the origin of the myofibroblast, the primary effector cell of peritoneal fibrosis, is not clearly established. Three hypotheses have been proposed regarding the cellular origin of the myofibroblast. The first, and historically most prevalent, hypothesis postulates that resident peritoneal fibroblasts respond to a variety of stimuli during fibrogenic responses and differentiate into myofibroblasts. The second hypothesis postulates that myofibroblasts are derived from bone marrow progenitor cells (7). A novel third possible source of fibroblasts and/or myofibroblasts in peritoneal fibrosis has recently been proposed: that human peritoneal mesothelial cells (HPMCs), through the process of epithelialmesenchymal transition (EMT), play a significant role (8).

EMT of epithelial cells, characterized by loss of epithelial cell characteristics and gain of ECM-producing myofibroblast characteristics, is an important mechanism involved in tissue fibrosis $(9,10)$. During parenchymal inflammation the HPMCs are exposed to a microenvironment with high levels of cytokines, chemokines and growth factors, including TGF- $\beta 1$ (8). TGF- $\beta 1$ is considered to be a master switch for the induction of fibrosis by a process of EMT in various organs including the peritoneum (11-13). During stress/injury HPMCs attain plasticity and lose their polarity and mesothelial markers. The cellular transition of HPMCs leads to cytoskeletal reorganization acquiring spindle-shape morphology and expression of mesenchymal markers. $\alpha$-smooth muscle actin ( $\alpha$-SMA) and vimentin are constitutively expressed in newly formed fibroblasts called myofibroblasts and are considered specific markers for EMT.

TGF- $\beta 1$ mediates EMT by inducing Smad signaling $(14,15)$. Smads are a group of intracellular proteins that are critical for transmitting the TGF- $\beta 1$ signals from the cell 
surface to the nucleus in order to promote transcription of target genes $(16,17)$. The role of Smad3 in the development of dermal fibrosis, subcapsular cataract, and peritoneal fibrosis has been reported $(18,19)$. However, the potential role of Smad2 in the development of fibrosis is unclear. In the present study we demonstrate in vitro that HPMCs undergo a transition from epithelial to mesenchymal phenotype in the presence of pro-fibrotic growth factor, TGF- 31 . The TGF- 31 induced HPMCs phenotypic transition to myofibroblasts is dependent on Smad2 signaling, thus suggesting the possibility that HPMCs may be the source of myofibroblasts in peritoneal fibrosis, and provide a favorable environment for the dissemination of gastric cancer.

\section{Materials and methods}

Cell line and culture. HPMCs were isolated from surgical specimens of human omentum as previously described (20). Briefly, small pieces of omentum were surgically resected under sterile conditions and trypsinized at $37^{\circ} \mathrm{C}$ for $30 \mathrm{~min}$. The suspension was then passed through a $200 \mu \mathrm{m}$ pore nylon mesh to remove undigested fragments and centrifuged at 2,000 rpm for $5 \mathrm{~min}$. The collected cells were cultured in RPMI-1640 supplemented with 10\% fetal calf serum (FCS). In the following experiments, cells were used during the second or third passage after primary culture. HPMCs were identified by immunostaining with mouse monoclonal antibodies against cytokeratin (Ck) and vimentin (DACO, Japan). The donors had no evidence of peritoneal inflammation and/or malignancy. All patients provided written informed consent prior to participation in the study. Our study was approved by the institutional ethics committee.

Phase contrast microscopy. The phenotypic changes of HPMCs were determined by phase contrast microscopy. The HPMCs in cultures were treated with recombinant TGF- $\beta 1$ (R\&D System, Minneapolis, MN) or left untreated (control) for 24 or $72 \mathrm{~h}$ and morphological changes were visualized by phase contrast microscopy. The images were collected using a Nikon inverted microscope (Nikon Corp., Japan).

Transmission electron microscopy. After incubation in test solutions for $24 \mathrm{~h}$, the cells were trypsinized and then fixed in ice-cold $2.5 \%$ electron microscopy grade glutaraldehyde in PBS ( $\mathrm{pH} 7.3$ ). The specimens were rinsed with PBS, post-fixed in $1 \%$ osmium tetroxide with $0.1 \%$ potassium ferricyanide, dehydrated through a graded series of ethanol (30-90\%), and embedded in Epon. Semithin (300 nm) sections were cut using a Reichart Ultracut, stained with $0.5 \%$ toluidine blue, and examined under a light microscope. Ultrathin sections $(65 \mathrm{~nm})$ were stained with $2 \%$ uranyl acetate and Reynold's lead citrate, and examined on a transmission electron microscope (Hitachi H-6001, Japan) at x5,000 magnification.

Western blot analysis for EMT markers. HPMCs were cultured on a 6-well tissue culture plate to confluence. The cells were treated with recombinant human TGF- $\beta 1$ once i.e. at the time of switching to serum-free medium, at a final concentration of 5 or $10 \mathrm{ng} / \mathrm{ml}$. HPMCs cultured without
TGF- $\$ 1$ were considered as control. The cells were harvested at 24,48 or $72 \mathrm{~h}$. Total cellular protein was extracted using a lysis buffer and quantified using protein quantification reagents from Bio-Rad. Next, $60 \mu \mathrm{g}$ of the protein were suspended in $5 \mathrm{X}$ reducing sample buffer, boiled for $5 \mathrm{~min}$, electrophoresed on $10 \%$ SDS-PAGE gels and transferred to polyvinylidene difluoride membrane by electroblotting. The membrane was blocked in $1 \% \mathrm{BSA} / 0.05 \%$ Tween/PBS solution overnight at $4^{\circ} \mathrm{C}$, followed by incubation with the primary antibody (mouse monoclonal antibodies to either human $\alpha$-SMA, vimentin, Ck, E-cadherin, fibronectin, collagen III, phosphorylated-Smad2, or Smad2) for $24 \mathrm{~h}$. A horseradish peroxidase-labeled goat anti-mouse IgG was used as the secondary antibody. The blots were then developed by incubation in a chemiluminescence substrate and exposed to X-ray film.

Reverse transcription polymerase chain reaction (RT-PCR). The cells were grown to subconfluence and then starved for $15 \mathrm{~h}$ in serum-free medium to attain quiescence. Afterwards, the cells were washed twice with PBS and cultured in either serum-free medium (control) or serum-free plus $5 \mathrm{ng} / \mathrm{ml}$ of TGF- 31 (experimental) for up to $72 \mathrm{~h}$. Total RNA was isolated from these cells using the TRIzol reagent according to the manufacturer's instructions. Total cellular RNA ( $1 \mu \mathrm{g})$ was then reverse-transcribed into cDNA for PCR amplification using a kit from Sigma. The primer sequences used for PCR are listed in Table I. Amplification consisted of an initial 5 min incubation at $95^{\circ} \mathrm{C}$ and then 30 cycles of amplification using $30 \mathrm{sec}$ of denaturation at $95^{\circ} \mathrm{C}, 30 \mathrm{sec}$ at $56^{\circ} \mathrm{C}$, and $60 \mathrm{sec}$ at $72^{\circ} \mathrm{C}$. The final extension was set for $10 \mathrm{~min}$ at $72^{\circ} \mathrm{C}$. All data were expressed as the relative differences between control and treated cells after normalization to $\beta$-actin expression.

Immunofluorescence staining. The expression of EMT markers in HPMCs was analyzed by immunofluorescence microscopy. In brief, the cells were cultured to confluence on collagen-coated glass cover slips and then fixed in $4 \%$ paraformaldehyde (with $50 \mathrm{mM}$ phosphate buffer) in $50 \%$ Tris wash buffer (TWB). The glass cover slips were rinsed three times and permeabilized with $1.2 \%$ Triton X-100 for $5 \mathrm{~min}$, rinsed three times, incubated with $1 \%$ BSA in $100 \%$ TWB for $1 \mathrm{~h}$, then stained for the expression markers using the following primary antibodies: mouse anti-E-cadherin (BD Transduction Laboratories, Franklin Lakes, NJ), mouse anti$\alpha$-SMA and mouse anti-fibronectin (Sigma) at 1:150 dilution; and respective secondary IgG antibodies conjugated with FITC or A546 (Zymax, San Francisco, CA). Cells were costained with TO-PRO 3 (blue) (Molecular Probes, Eugene, $\mathrm{OR}$ ) to visualize nuclei, were mounted with mounting medium and viewed by immunofluorescence microscopy.

Small interfering-RNA (siRNA) treatment. The HPMCs were grown to a $70 \%$ confluence on culture dishes and the transient transfection was performed with specific stealth siRNA against Smad2, or control siRNA overnight using Lipofectamine-2000 (Invitrogen; Carlsbad, CA), according the manufacturer's instructions. The total of three siRNA sequences for Smad2 and control-siRNA were designed and synthesized from Invitrogen using RNAi designer software 
Table I. Primers used for semi-quantitative RT-PCR.

\begin{tabular}{llc}
\hline Primer & \multicolumn{1}{c}{ Sequence } & Length (bp) \\
\hline Collagen III-F & 5'-GGACCACCAGGGCCTCGAGGTAAC-3' & 471 \\
Collagen III-R & 5'-TGTCCACCAGTGTTTCCGTG-3' & 451 \\
Fibronectin-F & 5'-TGGACCTTCTACCAGTGCGAC-3' \\
Fibronectin-R & 5'-TGTCTTCCCATCATCGTAACAC-3' \\
ß-actin-F & 5'-CCTCGCCTTTGCCGATCC-3' & 626 \\
ß-actin-R & 5'-GGATCTTCATGAGGTAGTCAGTC-3' \\
\hline
\end{tabular}
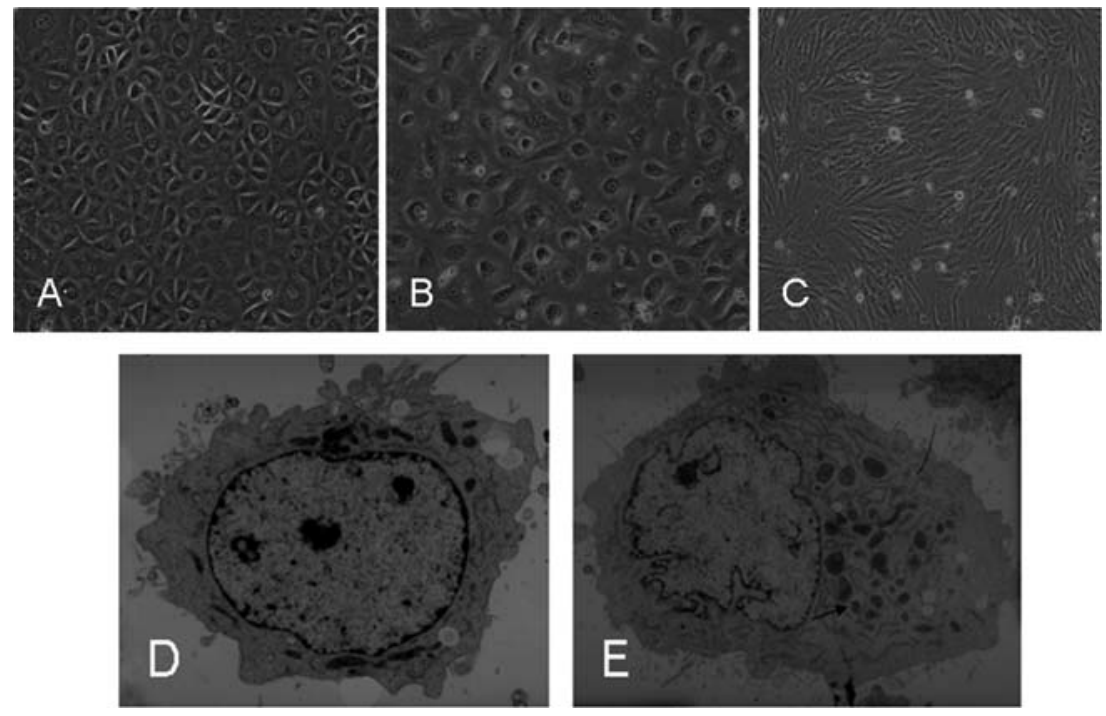

Figure 1. Phase contrast microscopy (A-C) and electron microscopy (D and E) of HMPCs. HPMCs were cultured in the presence ( $5 \mathrm{ng} / \mathrm{ml})$ or absence (control) of TGF- $\beta 1$ for $72 \mathrm{~h}$ in serum-free medium. The phenotypic changes (transition towards a myofibroblast-like phenotype) was evaluated by phase contrast microscopy and electron microscopy. (A) Control mesothelial cells without TGF-ß1 treatment exhibited epithelial cell morphology with a characteristic cobblestone-like growth pattern. (B) Few HPMCs converted to spindle fibroblast-like morphology at $24 \mathrm{~h}$. (C) The TGF-ß1-treated HPMCs showed transition to the more elongated morphological shape of myofibroblasts at $72 \mathrm{~h}$ (x40). (D) A representative electron micrographic montage of normal HPMCs, Mesothelial cells maintained a typical epithelial monolayer growth with scattered surface microvilli. (E) A representative electron micrographic montage of HPMCs after treatment with TGF- $\beta 1(5 \mathrm{ng} / \mathrm{ml})$ for 3 days. HPMCs showed characteristic myofibroblastic morphology with spindle multilayered growth and well-developed actin bundles decorated with dense bodies running through the cytoplasm (arrows). (x5,000).

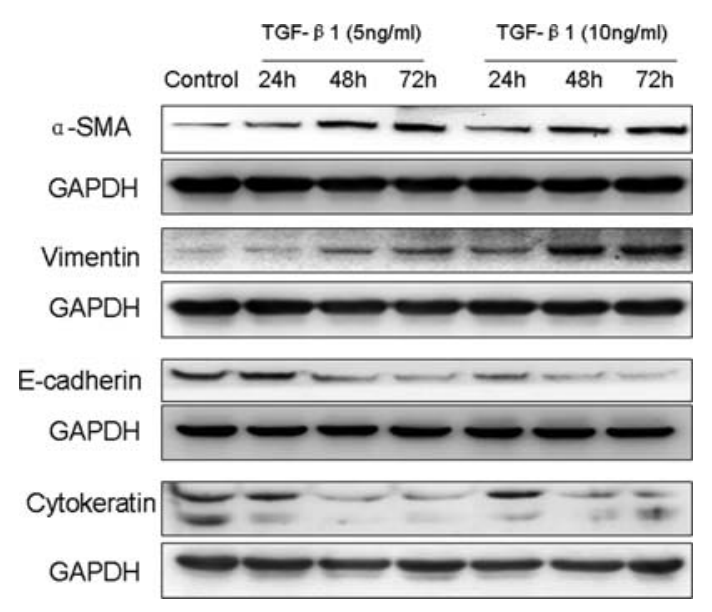

Figure 2. Expression of EMT markers in HPMCs in response to TGF-ß1 HPMCs were incubated in the presence or absence of TGF- $\beta 1$ for an indicated period of time. Whole protein cell lysates were immunoblotted with specific antibodies. TGF- $\beta 1$ treatment increased $\alpha$-SMA and vimentin expression after $72 \mathrm{~h}$ and decreased cytokeratin and E-cadherin expression after $48 \mathrm{~h}$ The blots were re-probed for GAPDH to ensure equal protein loading in each lane. Results are the representative data of three separate experiments. program. The concentration of $300 \mathrm{nM}$ was determined to be the most effective siRNA concentration for Smad2 silencing. The transfection medium was changed with culture medium containing 5\% FCS for $24 \mathrm{~h}$. TGF-B1 at a final concentration of $5 \mathrm{ng} / \mathrm{ml}$ was added to the cell cultures in serum-free medium or the cultures were left untreated (control). The cells were harvested at 4, 24 and $72 \mathrm{~h}$ for further experiments.

Statistical analysis. Data are expressed as mean \pm SD. Statistical comparisons of the data from the various groups were performed using the Student's t-test. Differences between groups were considered statistically significant at $\mathrm{P}<0.05$.

\section{Results}

TGF- $\beta 1$ induces mesenchymal phenotypic transformation in HPMCs. HPMCs were activated with recombinant TGF- 31 and morphological changes were observed after 24 and $72 \mathrm{~h}$. HPMCs cultured in serum-free medium in the absence of TGF-ß1 (control) showed a typical polygonal and cobblestone monolayer morphology (Fig. 1). HPMCs activated with 
Control
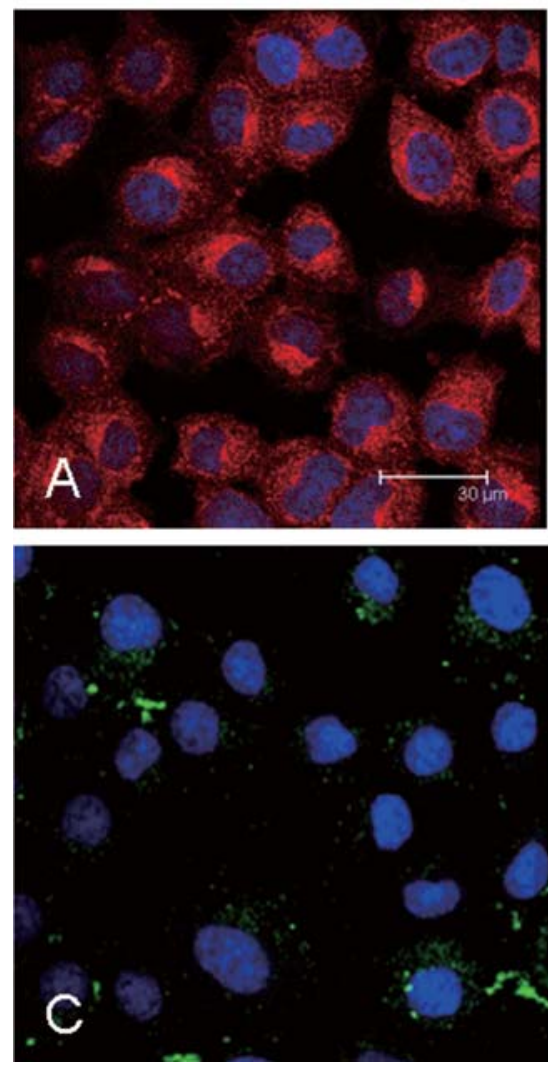

TGF- $\beta 1(5 \mathrm{ng} / \mathrm{ml}) 72 \mathrm{~h}$
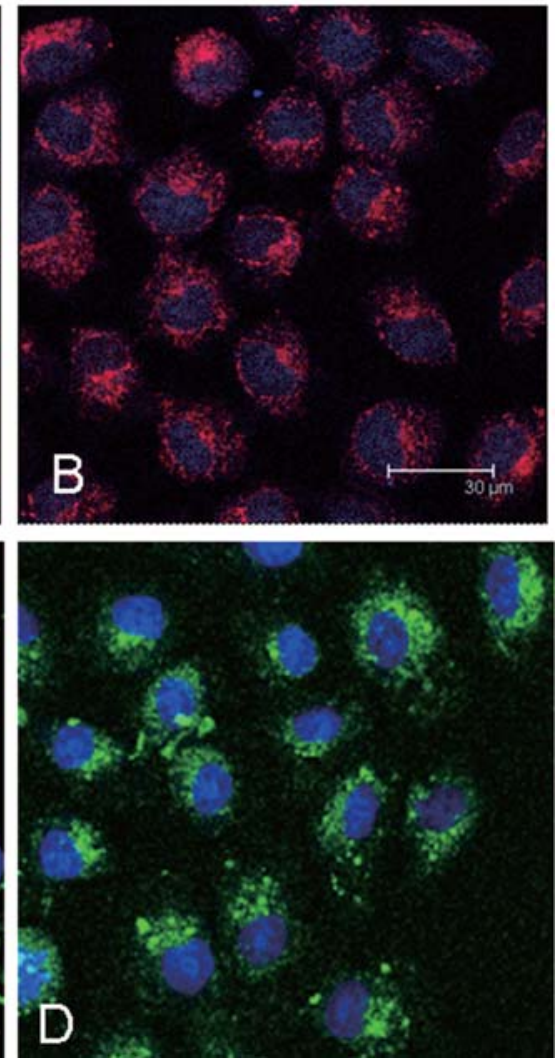

Figure 3. Immunofluorescence staining for E-cadherin (red) and $\alpha$-SMA (green) were evaluated by confocal laser microscopy. (B) There was apparent loss of cell membrane bound E-cadherin in TGF-ß1-treated HPMCs at $72 \mathrm{~h}$ compared to control (A). (D) The TGF-ß1-treated HPMCs exhibited a fibrillar pattern of $\alpha$-SMA-specific proteins at $72 \mathrm{~h}$. Also note that TGF- 31 caused spindle-shaped morphology, which was not observed in the control (C). The DNA dye TOPRO-3 (blue) was used for counterstaining. A546 (red) was used for E-cadherin and FITC (green) for $\alpha$-SMA. Scale bar, $30 \mu \mathrm{M}$.

TGF- $\$ 1$ showed phenotypic changes after $24 \mathrm{~h}$. Remarkable phenotypic changes were observed at $72 \mathrm{~h}$ of TGF- $\beta 1$ activation. When compared to control, TGF- $\beta 1$ activated HPMCs showed elongated, spindle-shaped morphology, characteristic of fibroblasts suggesting that HPMCs are capable of expansion and differentiation into a different phenotype. The treatment of HPMCs with TGF- 31 showed characteristic myofibroblastic morphology with spindle multilayered growth and well-developed actin bundles decorated with dense bodies running through the cytoplasm.

TGF- $\beta 1$ induces the expression of EMT markers in HPMCs. TGF-B1-induced phenotypic changes in HPMCs were evaluated by assessing the expression of epithelial and mesenchymal markers. HPMCs were activated with TGF- 31 for 24 , 48 or $72 \mathrm{~h}$ or left untreated. We noted the down-regulation of epithelial markers in TGF-ß1-activated HPMCs. This was accompanied by up-regulation of mesenchymal markers. E-cadherin and $\mathrm{Ck}$ are epithelial markers and maintain the cellular integrity. Down-regulation of E-cadherin and $\mathrm{Ck}$ mark the onset of EMT. The expression of E-cadherin and $\mathrm{Ck}$ was significantly decreased in TGF- 11 -treated HPMCs when compared to control (Fig. 2). To demonstrate the HPMCs transition into myofibroblast we also examined the expression of $\alpha$-SMA, a phenotypic marker for myofibroblast cells. An increase in the expression of $\alpha$-SMA was noted after $24 \mathrm{~h}$ and persisted at $72 \mathrm{~h}$ when compared to control
Control $15 \min 30 \min 60 \min 3 \mathrm{~h} \quad 24 \mathrm{~h}$

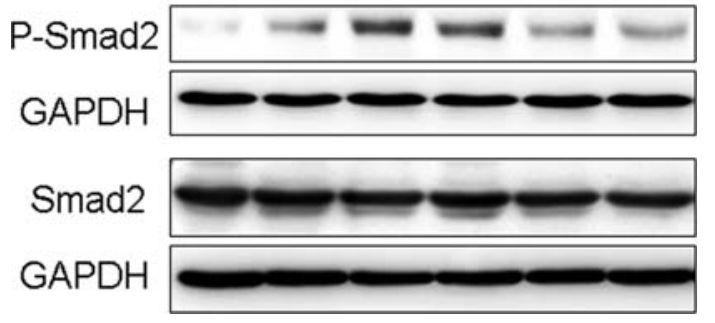

Figure 4. TGF- $\beta 1$ induces Smad 2 signaling in HPMCs. HPMCs were incubated in the presence of TGF- $\beta 1(5 \mathrm{ng} / \mathrm{ml})$ for an indicated period of time or left untreated. Whole cell lysates were obtained and analyzed for the phosphorylated forms of Smad2 (P-Smad2) and for Smad2. An increase in P-Smad2 was noted up to $30 \mathrm{~min}$ and it decreased by $24 \mathrm{~h}$. Expression of Smad2 remained unchanged in control HPMCs and TGF-B1-treated HPMCs. GAPDH was used as a loading control to demonstrate equal protein loading. Results are the representative of three separate experiments.

HPMCs. The relative protein expression of vimentin, a specific marker for newly formed myofibroblasts, increased over time (Fig. 2).

Immunofluorescence staining for E-cadherin and $\alpha$-SMA expression. In addition to Western blotting, the expression of E-cadherin and $\alpha$-SMA were evaluated in resting and TGF- $\beta 1-$ treated HPMCs by immunofluorescence. We noted strong 
A

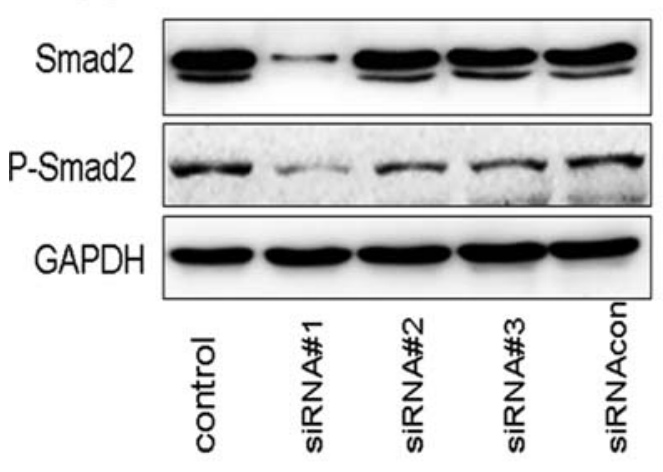

B
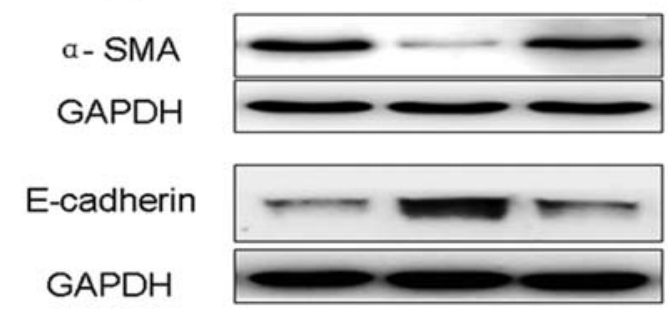

SiRNA-Smad2 - $\quad+\quad-$

Control-siRNA $-\quad-\quad+$

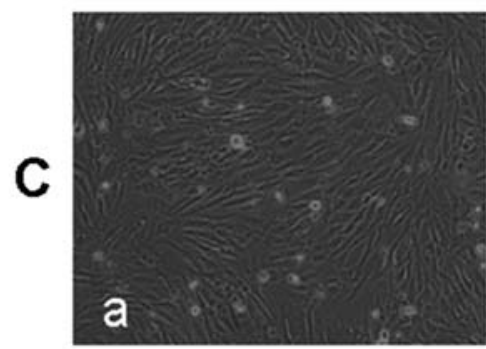

TGF- $\beta 1$

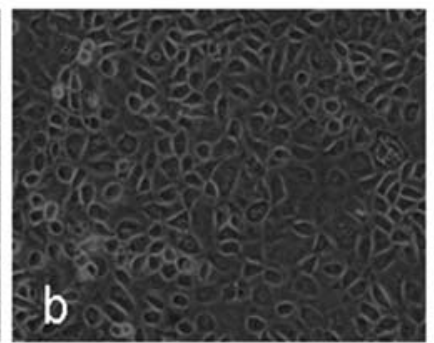

TGF- $\beta 1+$ siRNA

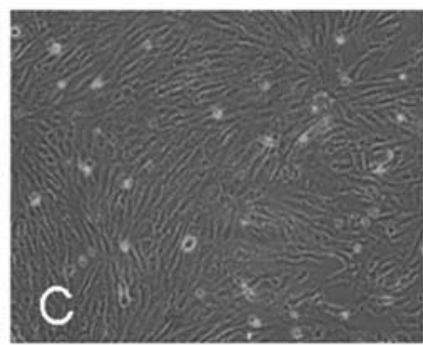

TGF- $\beta 1+$ siRNA-Con

Figure 5. Smad2 siRNA suppresses the expression of Smad2 and the morphological changes in TGF-ß1-activated HPMCs. (A) HPMCs were transfected with siRNA Smad2, activated with TGF-B1 for $4 \mathrm{~h}$ and the transfection efficiency was confirmed. Three specific siRNAs for Smad2 along with a control siRNA were used. siRNAi-Smad2 \#1 showed a highly significant knockdown for Smad2 and phosphorylated Smad2 (P-Smad2) when compared to other two siRNAs or to the control siRNA. (B) HPMCs were transfected with siRNA-Smad2 or control siRNA and treated with TGF- 31 for 72 h. Smad2 siRNA transfection preserves E-cadherin expression and suppresses $\alpha$-SMA in TGF- $\beta 1$-treated HPMCs. (C) Phase contrast microscopy of HPMCs exposed to TGF- $\beta 1$ alone or transfected with siRNA-Smad2. The phenotypic changes (transition towards a myofibroblast-like phenotype) were clearly evident after $72 \mathrm{~h}$. Smad2 siRNA but not control siRNA preserved the cobblestone morphology of HPMCs.

expression of E-cadherin in resting HPMCs. However, the expression of E-cadherin was significantly down-regulated in TGF- 31 treated HPMCs. The expression of $\alpha$-SMA was also determined by immunofluorescence staining. Strong expression of $\alpha$-SMA was noted in HPMCs after $48 \mathrm{~h}$ of treatment with TGF-ß1 (Fig. 3).

TGF- $\beta 1$ treatment induces Smad2 phosphorylation in HPMCs undergoing phenotypic transformation. We examined the expression of Smad2 in HPMCs treated with TGF- $\beta 1$ and in resting HPMCs. TGF- $\beta 1$ ( $5 \mathrm{ng} / \mathrm{ml})$ induced phosphorylation of Smad2 within $10 \mathrm{~min}$ of stimulation, and the level of Smad2 phosphorylation reached a maximum between 30-60 min after treatment and remained elevated for the duration of the experiment without affecting total Smad2 expression (Fig. 4). Taken together, these data indicate that rapid and sustained phosphorylation of Smad2 is associated with TGF-ß1-induced EMT events.

Silencing Smad2 signaling blocks TGF- $\beta 1$ induced mesenchymal transformation in HPMCs. In order to confirm whether Smad2 is involved in TGF- $\beta 1$ mediated EMT in HPMCs, siRNAs were used to knock down Smad2 gene in HPMCs. We first evaluated the expression of E-cadherin and $\alpha$-SMA in total cell lysates by Western blot analysis, after silencing Smad2 by using siRNA-Smad2 or control-siRNA in HPMCs treated with TGF- $\beta 1$. We noted a remarkably reduced expression of $\alpha-\mathrm{SMA}$, and most importantly a significant restoration of the junctional protein E-cadherin suggesting a role for Smad2 signaling in EMT of HPMCs (Fig. 5B). We also confirmed the effect of siRNA-Smad2 on HPMCs treated with TGF- 11 by phase contrast microscopy. As shown in Fig. 5C, the cellular morphology of TGF-ß1treated HPMCs was reverted from a spindle shape to a more cuboidal/cobblestone shape, after Smad2 knockdown. This effect was not observed in the control siRNA-treated HPMCs in the presence of TGF- 1 . This suggests that the mesenchymal changes induced by TGF- $\beta 1$ in HPMCs are regulated by Smad2 signaling.

TGF- $\beta 1$ induces collagen III and fibronectin synthesis in HPMCs. To further evaluate our hypothesis whether the phenotypic changes noted after treatment of HPMCs with TGF-B1 was due to ECM protein synthesis, we examined the expression of collagen III and fibronectin at the transcriptional and translational levels in HPMCs activated with TGF-ß1. A significantly increased synthesis of collagen III and fibronectin ware noted after TGF- $\beta 1$ treatment. Treatment of HPMCs with siRNA-Smad2 prior to TGF- 1 stimulation significantly inhibited the expression of collagen III and fibronectin compared to control. We also examined the expression of fibronectin by immunofluorescence staining. Fibronectin fibrils were clearly detected in the cytoplasmic region of HPMCs treated with TGF- 31 at $72 \mathrm{~h}$, but HPMCs transfected with siRNA-Smad2 showed some fibronectin deposition (Fig. 6). 
A

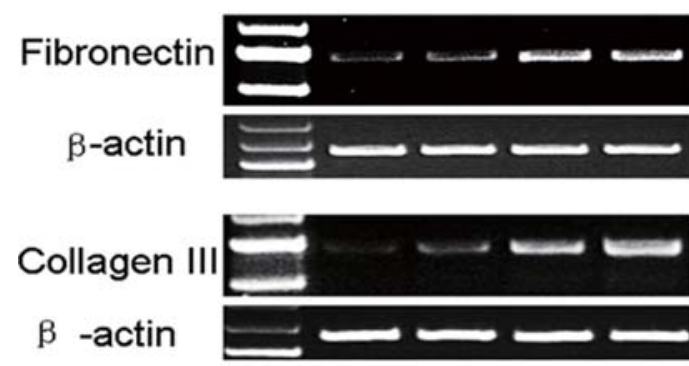

TGF- $\beta 1-\quad+\quad+\quad+$

SIRNA $-\quad+\quad-\quad-$ SiRNA-con $\quad-\quad-\quad+\quad-$
B

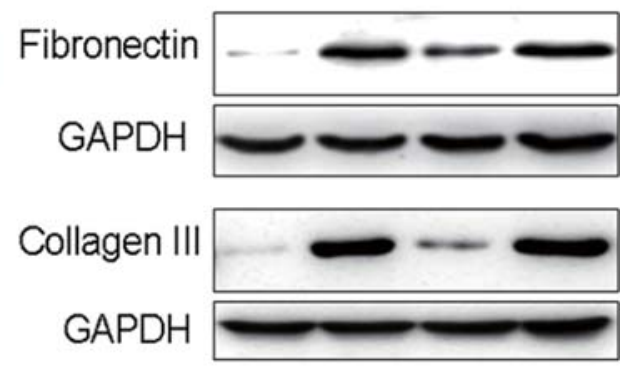

TGF- $\beta 1-\quad+\quad+\quad+$

SIRNA - $\quad-\quad+\quad-$

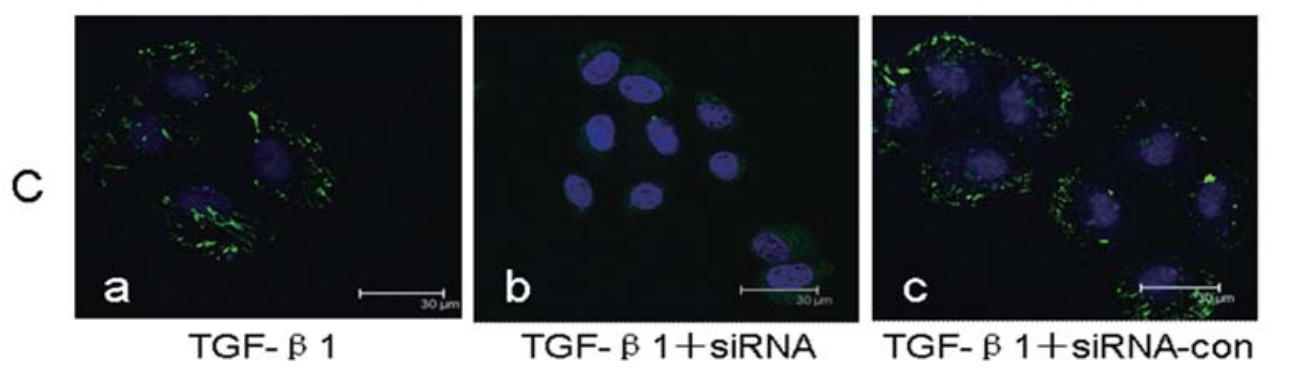

Figure 6. Collagen III and fibronectin mRNA and protein levels in HPMCs. (A) The mRNA expression of collagen III and fibronectin was evaluated by RT-PCR. HPMCs were transfected with siRNA-Smad2 or control siRNA and treated with TGF-ß1 for 72 h. Smad2 siRNA transfection suppresses collagen III and fibronectin mRNA in TGF-ß1 treated HPMCs. (B) Western blot analysis of collagen III and fibronectin protein levels in HPMCs. HPMCs were transfected with siRNA-Smad 2 or control siRNA and treated with TGF- 31 for $72 \mathrm{~h}$. Smad 2 siRNA transfection suppresses collagen III and fibronectin protein expression in TGF-ß1-treated HPMCs. (C) Immunofluorescence staining for fibronectin (green) was evaluated by fluorescence microscopy. TGF-ß1-treated HPMCs upregulated fibronectin expression. HPMCs transfected with siRNA-Smad2 showed some fibronectin deposition. Scale bar, $30 \mu \mathrm{M}$.

\section{Discussion}

In this study we demonstrate that HPMCs respond to TGF- $\beta 1$ by transformation into myofibroblasts in vitro indicating that these cells have the capacity to initiate and modulate the expression of epithelial markers to mesenchymal markers to undergo EMT. Because HPMCs can transform into a myofibroblast-like phenotype and secrete ECM compounds in response to TGF- $\beta 1$, it is possible that HPMCs play a role during peritoneal fibrosis and the peritoneal dissemination of gastric cancer. A growing body of evidence indicates that HPMCs undergo EMT during peritoneal dialysis and that EMT play a key role in the development of peritoneal fibrosis $(8,13,15)$. Our previous study demonstrated that TGF- $\beta 1$ levels in peritoneal lavage fluid are significantly correlated with peritoneal metastasis and TNM stages of gastric cancer (21). The basal surface of mesothelial cells is bathed in epithelial lining fluid which may contain cytokines/ chemokines. It is plausible that local parenchymal inflammation induced by the cytokine milieu may lead to disruption of peritoneal mesothelial homeostasis. A key feature of peritoneal dissemination of gastric cancer is the presence of high levels of TGF- $\beta 1$ in ascitic fluid (21). The continuous exposure of mesothelial cells to high levels of the cytokine TGF- $B 1$ causes injury and may promote a mesenchymal phenotype.

The growth factor TGF- 11 contributes to the development of fibroblastic foci in the sub-peritoneal region, and several studies have focused on the role of TGF- 31 as the key promoter of peritoneal fibrosis $(19,22)$. However, the precise mechanisms responsible for the formation of fibroblastic foci are unknown and the origin of myofibroblasts, critical elements in the process of fibrosis is not clearly understood. It is recognized that injury of the peritoneal mesothelium precedes the formation of peritoneal fibroblasts and HPMCs may therefore contribute to their formation, through the production of key fibrogenic mediators, including TGF- 31 (23). Our data demonstrate that HPMCs undergo transition from the epithelial to the mesenchymal phenotype upon activation with TGF- 31 , with the induction of the transcription factor Smad2 and a dramatic down-regulation of $\mathrm{Ck}$ and of the junctional protein E-cadherin expression.

Smad-independent and -dependent signaling is involved in the onset of EMT. Recent findings demonstrated that Smad3 is involved in TGF- 31 -induced peritoneal fibrosis and have indicated that Smad3 is essential for EMT (15). In the present study, we have demonstrated the role of Smad2 in TGF-ß1 mediated EMT in HPMCs. The significance of the present study is that HPMCs undergo the process of EMT via expression of the mesenchymal markers vimentin and $\alpha$-SMA, and siRNA-Smad2 significantly blocked the expression of $\alpha$-SMA in HPMCs activated with TGF- $\beta 1$ and prevented EMT in HPMCs.

In order to confirm the effect of EMT on peritoneal fibrosis, we showed that TGF- $\beta 1$ affected the function of mesothelial cells by stimulating ECM (including fibronectin 
and collagen III) production, but Smad2 siRNA transfection suppresses collagen III and fibronectin protein expression in TGF-ß1-treated HPMCs. Meanwhile, immunolocalization showed that expression of fibronectin protein was induced by TGF- 11 in HPMCs, while HPMCs transfected with siRNASmad2 did show some fibronectin deposition. These data further support the central role theory for EMT in peritoneal fibrosis and may provide a useful model by which to study peritoneal metastasis of gastric cancer.

In conclusion, HPMCs could be the potential origin of myofibroblasts and may in part contribute to peritoneal fibrosis. Studies on signaling mechanisms, cytokines and their receptors in HPMCs could shed light on the mechanisms involved in fibrotic disease and the peritoneal dissemination of gastric cancer.

\section{Acknowledgements}

This study was supported by the National Natural Science Foundation of China (nos. 30672050, 30873043 and 81071956). We thank Professor Feng Li for technical assistance and Dr Jiamei Wu and Dr Chunyu Wang, Dr Qiang Ke, Dr Jian Zhang and Dr Shuo Wang for precious advice.

\section{References}

1. Sugarbaker PH and Yonemura Y: Clinical pathway for the management of resectable gastric cancer with peritoneal seeding: best palliation with a ray of hope for cure. Oncology 58: 96-107, 2000

2. Otsuji E, Kuriu Y, Okamoto K, Ochiai T, Ichikawa D, Hagiwara A and Yamagishi H: Outcome of surgical treatment for patients with scirrhous carcinoma of the stomach. Am J Surg 188: 327-332, 2004.

3. Ahmed N, Riley C, Rice G and Quinn M: Role of integrin receptors for fibronectin, collagen and laminin in the regulation of ovarian carcinoma functions in response to a matrix microenvironment. Clin Exp Metastasis 22: 391-402, 2005.

4. Lessan K, Aguiar DJ, Oegema T, Siebenson L and Skubitz AP: CD44 and beta1 integrin mediate ovarian carcinoma cell adhesion to peritoneal mesothelial cells. Am J Pathol 154: 1525-1537, 1999.

5. Krzysztof KZ, Justyna MP and Katarzyna K: Senescent peritoneal mesothelial cells promote ovarian cancer cell adhesion. Am J Pathol 174: 1231-1240, 2009.

6. Yashiro M, Chung YS, Nishimura S, Inoue $\mathrm{T}$ and Sowa $\mathrm{M}$ : Fibrosis in the peritoneum induced by scirrhous gastric cancer cells may act as 'soil' for peritoneal dissemination. Cancer 77: S1668-S1675, 1996.

7. Epperly MW, Guo H, Gretton JE and Greenberger JS: Bone marrow origin of myofibroblasts in irradiation pulmonary fibrosis. Am J Respir Cell Mol Biol 29: 213-224, 2003.
8. Yanez-Mo M, Lara-Pezzi E, Selgas R, Ramirez-Huesca M, Dominguez-Jimenez C, Jimenez Heffernan JA, Aguilera A, Sanchez-Tomero JA, Bajo MA, Alvarez V, Castro MA, del Peso G, Cirujeda A, Gamallo C, Sanchez-Madrid F and LopezCabrera M: Peritoneal dialysis and epithelial-to-mesenchymal transition of mesothelial cells. N Engl J Med 348: 403-413, 2003.

9. Xu J, Lamouille S and Derynck R: TGF-ß-induced epithelial to mesenchymal transition. Cell Res 19: 156-172, 2009.

10. Imamichi Y and Menke A: Signaling pathways involved in collagen-induced disruption of the E-cadherin complex during epithelial-mesenchymal transition. Cells Tissues Organs 185: 180-190, 2007.

11. Friedman SL: Molecular regulation of hepatic fibrosis, an integrated cellular response to tissue injury. J Biol Chem 275: 2247-2250, 2000.

12. Verrecchia F and Mauviel A: Transforming growth factor-beta and fibrosis. World J Gastroenterol 13: 3056-3062, 2007.

13. Kalluri R and Neilson EG: Epithelial-mesenchymal transition and its implications for fibrosis. J Clin Invest 112: 1776-1784, 2003.

14. Kaimori A, Potter J, Kaimori JY, Wang C, Mezey E and Koteish A: Transforming growth factor-beta 1 induces an epithelial-to-mesenchymal transition state in mouse hepatocytes in vitro. J Biol Chem 282: 22089-22101, 2007.

15. Liu Q, Mao H, Nie J, Chen W, Yang Q, Dong X and Yu X. Transforming growth factor \{beta\} 1 induces epithelialmesenchymal transition by activating the JNK-Smad3 pathway in rat peritoneal mesothelial cells. Perit Dial Int 3: S88-S95, 2008.

16. Shi Y and Massague J: Mechanisms of TGF-beta signaling from cell membrane to the nucleus. Cell 113: 685-700, 2003.

17. Dijke P and Hill CS: New insights into TGF-beta-Smad signalling. Trends Biochem Sci 29: 265-273, 2004.

18. Shirai K, Saika S, Tanaka T, Okada Y, Flanders KC, Ooshima A and Ohnishi Y: A new model of anterior subcapsular cataract: involvement of TGFbeta/Smad signaling. Mol Vision 12: 681-691, 2006.

19. Schiller M, Javelaud D and Mauviel A: TGF-beta-induced SMAD signaling and gene regulation: consequences for extra-cellular matrix remodeling and wound healing. J Dermatol Sci 35: 83-92, 2004.

20. Oravecz T, Pall M, Roderiquez G, Gorrell MD, Ditto M, Nguyen NY, Boykins R, Unsworth E and Norcross MA: Regulation of the receptor specificity and function of the chemokine RANTES (regulated on activation, normal T cell expressed and secreted) by dipeptidyl peptidase IV (CD26)mediated cleavage. J Exp Med 186: 1865-1872, 1997.

21. Su XH, You ZY and Xu HM: Relationship between the pathology and TGF- $\beta 1$ presented in gastric cancer and peritoneal lavage fluid. J China Med University 34: 164-166, 2005.

22. Verrecchia $\mathrm{F}$ and Mauviel A: Transforming growth factor-beta signaling through the Smad pathway: role in extracellular matrix gene expression and regulation. J Invest Dermatol 118: 211-215, 2002 .

23. Na D, Lv ZD, Liu FN, Xu Y, Jiang CG, Sun Z, Miao ZF, Li F and $\mathrm{Xu} H \mathrm{HM}$ : Transforming growth factor $\beta 1$ produced in autocrine/paracrine manner affects the morphology and function of mesothelial cells and promotes peritoneal carcinomatosis. Int J Mol Med 26: 325-332, 2010. 